\title{
Special issue on cognitive enhancers
}

\author{
T. W. Robbins
}

Received: 20 October 2008 / Accepted: 20 October 2008 / Published online: 11 November 2008

(C) Springer-Verlag 2008

In the 1970s, a classic text-book (Iversen and Iversen 1975) came to the conclusion that: "The cellular and biochemical changes associated with learning and memory are largely unknown". However, the same authors pointed out optimistically that "the unsatisfactory state of knowledge in this area does not mean that drug effects on the processes of learning and memory cannot be studied" (p186).

We have learned much about the molecular, cellular and neuropsychological bases of learning and memory over the intervening 30 years or so, and there is thus even more of a rationale for studies of memory-enhancing drugs and for drugs that similarly boost several other aspects of cognitive function. The vibrancy of the field can be gauged from the enthusiastic response to the call for contributions to this Special Issue. The term 'nootropic', meaning cognitive enhancing drug (or 'smart' drug) was coined in 1964 by C. Giurgea (nootropic comes from the Greek words: noos = mind and tropein $=$ to bend or turn). The term was originally applied rather narrowly to the group of drugs typified by the pyrrolidone, piracetam, which had a range of neurochemical effects including the enhancement of brain cell energy and metabolism and which had provided promising signals on simple rodent tests of learning and memory, generally derived from the memory consolidation literature, including the post-trial administration paradigm. Today, drugs such as hydergine, which work on roughly similar principles, are being used in the treatment of dementia in the elderly. Meanwhile, an alternative approach for the treatment of dementia was being formulated based

\section{T. W. Robbins $(\triangle)$}

Department of Expt. Psychology and Behavioural and Clinical Neuroscience Institute, University of Cambridge,

Cambridge CB2 3EB, UK

e-mail: twr2@hermes.cam.ac.uk on the model of cholinergic repletion, using the spatial delayed response task in primates, which was later accepted as a test of human 'working memory', in what was one of the first, though sustained, translational initiatives. The spatial delayed response task and its analogues were shown to be susceptible to muscarinic receptor blockade in both non-human primates and in humans, for example, suffering from Alzheimer's disease. Given these traditions, it is especially appropriate that this Special Issue includes Reviews by two of the originators of the field, JL McGaugh and RT Bartus. Roozendaal and McGaugh provide a twenty-first-century re-appraisal of memory consolidation, focusing on the neurochemical modulation of amygdaloid function, with possible implications for the treatment of aberrant 'emotional memories', as occur for example in post-traumatic stress disorder. The complementary Review by Bartus and Dean covers their original seminal contributions to the cholinergic hypothesis of geriatric memory dysfunction and how this hypothesis has shaped subsequent research leading to drugs such as donepezil. Alzheimer's disease is a good example of where the strategies for achieving cognitive enhancement have varied mechanistically from neuroprotection (e.g. beta and gamma secretase inhibitors) to symptomatic treatments that depend on boosting the functioning of deteriorating neural circuits (e. g. with nicotine, 5-HT6 receptor antagonists, galantamime, $\mathrm{H} 3$ receptor agents, and even via herbal medicines such as Gingko).

There have, of course, been many other major influences on the field - for example, the discovery of the glutamate receptor subtypes and their roles in learning and memory, as well as neuronal models of learning such as long-term potentiation. It has become clear that glutamate contributes to the effective operation of cortical circuitry in higher cognitive processes and that $\mathrm{N}$-methyl-D-aspartate receptor 
function may be deficient in schizophrenia, being associated with the considerable evidence of cognitive impairment in this disorder (reviews by Galletly and Sarter et al.). Therefore, various means of boosting glutamatergic transmission have come to the fore in the search for enhancing cognition, including glycine uptake inhibitors and alphaamino-3-hydroxy-5-methyl-4-isoxazolepropionate-receptor modulators ('Ampa-kines'). A key issue in schizophrenia research has thus become whether cognitive deterioration can be avoided either by the design of new anti-psychotic agents with fewer side effects or by the discovery of possible 'add-on' treatments to enhance cognition directly. Needless to say, there are several other strategies being pursued in the remediation of 'fronto-executive' deficits in schizophrenia, including subtype-selective nicotinic receptor agonists and dopamine D1 receptor signalling.

Yet another strand in research in cognitive enhancement has derived from the efficacy of drugs such as methylphenidate in the treatment of attention deficit/hyperactivity disorder (ADHD), presumably in part as a consequence of its actions on fronto-striatal systems. Despite this relative success, it is still not quite clear how such drugs actually produce their therapeutic effects. For example, is it the modulation of striatal dopamine or either cortical noradrenaline or dopamine function that is key? This has also led to a strategy for discovering new 'atypical stimulants' such as atomoxetine, clozapine and modafinil which only have part of the psychomotor stimulant profile of methylphenidate.

The catecholaminergic modulation of fronto-executive functions has long been known to depend on a YerkesDodson relationship, whereby cognitive function is best performed at an optimal level of catecholaminergic function (e.g. Arnsten 1998). But the field has also advanced in terms of a fresh focus, not on neurotransmitters and receptors per se but upon secondary and tertiary molecules and biochemical cascading pathways - this is surely a hint of future approaches to drug discovery and design in this area. This theme is exemplified by reviews on protein kinase inhibitors (review by Arnsten), phosphodiesterase inhibitors (review by Reneerkens et al.) and even lithium (review by Tsaltas et al.), for which recent fundamental discoveries have implicated specific intracellular cascades in its actions which may well include plasticity and consequent cognitive improvement in certain situations.

Readers of the various articles in this issue will realise two major general points - first the increasing sophistication at the behavioural as well as the pharmacological levels as translational divides between species are becoming partly bridged (e.g. Ko and Evenden) - including examples of effective 'back-translation' (Dias et al.) and also the wide variety of cognitive syndromes that may be amenable to medication by cognitive enhancing drugs, ranging from brain injury to Alzheimer's disease, depression, ADHD and schizophrenia.

A further consequence, much seized upon by the media, comes from the finding that healthy human subjects may also be subject to 'cognitive enhancement'. Although this has sometimes been a difficult concept to grasp for some, it is clear that states such as fatigue, stress, mood and sleep deprivation all powerfully impact on cognition, in a manner predicted by the Yerkes-Dodson relationship. However, a further derivation from this principle is that not all aspects of cognition are likely to be improved by medication. Indeed, some are likely to improve at the costs of others, given the theoretical difficulty of optimising all aspects of a complex system and the need to recruit cognitive resources appropriate to the context at hand (see Farah et al.). As the UK Government 'Technology Foresight' initiative has noted (Jones et al. 2007), we will have to give some attention to the costs of using such cognitive enhancers when assessing their benefits (see also Academy of Medical Sciences Working Group Report, 2008). The use of cognitive enhancing drugs for non-medical purposes has also attracted neuroethical debate (Sahakian and MoreinZamir 2007), but this is beyond our present scope. The theme of this Special Issue is critically to survey the objective evidence for cognitive enhancement via drugs affecting a range of different neurobiological mechanisms.

\section{References}

Academy of Medical Sciences U.K. Working Group Report on Brain science, addiction and drugs (2008) Chapter 8 Cognition Enhancers, pp 143-160

Arnsten AFT (1998) Catecholamine modulation of prefrontal cortical function. Trends Cogn Sci 2:436-447

Iversen SD, Iversen LL (1975) Behavioral pharmacology. Oxford University Press, New York

Jones R, Morris K, Nutt D (2007) Cognition enhancers, Chapter 8. In: Nutt D, Robbins TW, Stimson GV, Ince M (eds) Drugs and the future: brain science, addiction and society. Elsevier, Amsterdam, pp 241-283

Sahakian BJ, Morein-Zamir S (2007) Professor's little helper. Nature 450:1157-1159 